\title{
PROJETO E DESENVOLVIMENTO DE UM CONTROLADOR INDUSTRIAL APLICADO A UM SISTEMA TERMOELÉTRICO DE BAIXO CUSTO
}

\author{
DOI: 10.37702/2175-957X.COBENGE.2021.3430
}

Caio Cesar Jacob Silva - caiocjacob@gmail.com

Instituto Federal de São Paulo

Avenida Salgado Filho 3501

07115-000 - Guarulhos - SP

Marcos Henrique Nunes Salvador - marcos.salvador@aluno.ifsp.edu.br

Instituto Federal de São Paulo

Praça 31 de Março 162

06194-070 - Osasco - SP

Tarcísio Fernandes Leão - leao@ifsp.edu.br

Instituto Federal de São Paulo IFSP

Avenida Estevão de Albuquerque 25

04419-120 - São Paulo - SP

Resumo: Este trabalho visa facilitar o processo de ensino-aprendizagem das disciplinas de teoria de controle aplicando, em uma planta didática de simples construção e baixo custo, técnicas de identificação, modelagem, projeto e sintonia de controladores industriais. O sistema termoelétrico foi projetado com componentes discretos em conjunto com a plataforma Arduino e a malha de controle foi implementada com o auxílio do software MATLAB/Simulink. A planta possui um atraso de propagação, o que possibilita a utilização de técnicas de sintonia para o controlador PID menos comuns, como o método de Cohen-Coon. Os resultados mostraram que a identificação do sistema e a sintonia do controlador foram satisfatórias, pois a temperatura dos modelos e da planta física apresentaram um comportamento estável em regime permanente.

Palavras-chave: Controle de processos, controlador PID, planta didática, sistema termoelétrico. 


\section{INTRODUÇÃO}

É perceptível a dificuldade que as pessoas têm em compreender um determinado tema quando ele é tratado somente de forma teórica. Com os alunos da área de exatas o problema se torna ainda mais visível, pois vários assuntos podem ser considerados abstratos quando não há uma metodologia experimental.

As aulas práticas buscam fixar o conteúdo aprendido em sala de aula, complementando os conteúdos teóricos, facilitando a compreensão, tornando o conteúdo mais interessante e agradável e possibilitando ao aluno o desenvolvimento de habilidades de observação, análise e resolução de problemas (DIXON et. al, 2012).

O objetivo principal desse artigo é facilitar o processo de ensino-aprendizagem das disciplinas de teoria de controle, usando uma planta didática de simples construção. $O$ sistema termoelétrico é desenvolvido com componentes discretos de baixo custo, propiciando aos alunos a aquisição de dados, a identificação de modelos matemáticos e a aplicação e sintonia de controladores.

Um sistema de controle é um conjunto de componentes interconectados que tem como objetivo fazer o sistema a ser controlado produzir uma saída desejada. São aplicados em diversas áreas, como transportes e navegação, medicina, astronomia, entre outros, e têm se tornado parte essencial dos processos industriais (OGATA, 2003).

Com a implementação da planta didática é possível representar, de forma simples e objetiva, os parâmetros de um sistema de controle com tempo morto, realizar o estudo das técnicas de controle em modelos teóricos e comparar sua resposta com o sistema físico.

\section{FUNDAMENTAÇÃO TEÓRICA}

Os cursos de engenharia têm um caráter prático, assim a utilização de laboratórios é de grande importância na formação do estudante. Porém sua utilização enfrenta alguns problemas, como os custos de aquisição dos equipamentos, sua correta manutenção e operação. Assim, faz-se necessário a busca por soluções alternativas, como o desenvolvimento de equipamentos de baixo custo como ferramenta de ensino (GOMES et. al, 2011).

\subsection{Controle PID}

Há diversas técnicas de controle aplicáveis em um determinado sistema. No caso de sistemas mais simples, onde suas variáveis de entrada assumem apenas dois valores distintos, sendo eles zero ou o valor desejado, em que não há a necessidade de controle estável e exato e a frequência de oscilação é baixa, ou seja, o processo é lento, é muito utilizado o controle On-Off. Porém, como esse tipo de controle pode gerar muitos ruídos na saída, quando esses interferem no resultado, o uso do controlador Proporcional Integral Derivativo (PID) passa a se tornar uma técnica mais adequada por permitir a seleção e controle de diversos valores, apresentando como resultado um controle mais preciso desse sistema.

Segundo Garcia (2017) a função de um controlador é manter a variável controlada no valor mais próximo possível do valor desejado, independentemente das variações da carga de alimentação ou de demanda. Devido a sua versatilidade, facilidade de implementação e apresentar resultados satisfatórios, o controlador PID é o algoritmo de controle mais utilizado.

Em resumo, no controlador PID, a composição de proporcionalidade tem por função efetuar a correção proporcional ao erro, a correção a ser aplicada ao processo deve crescer 
na proporção que cresce o erro entre o valor real e o setpoint. A composição integrativa não pode ser empregada de forma isolada, pois ela tem o efeito de eliminar o desvio característico de um controle puramente proporcional. A ação integral atua corrigindo o valor da variável manipulada em intervalos regulares, somando a esta o valor do desvio entre a variável manipulada e o setpoint, denominando assim estes intervalos de atuação como tempo integral, ou expresso pelo seu inverso, chamado de taxa integral, sendo a ação integral responsável por eliminar o erro em regime permanente, porém não poderá haver uma atuação excessiva para que não ocorra uma instabilidade no sistema. Partindo pela mesma lógica, a ação derivativa tem o efeito de reduzir a velocidade das variações que são controladas no processo, evitando que se eleve ou reduza muito rapidamente, agindo somente quando há uma variação no erro, logo, durante as perturbações ou início do processo, quando há um erro no sistema, a componente derivativa age de forma a atenuar as variações, melhorando o desempenho do sistema durante os transitórios (Novus, 2003).

Assim temos que a Equação (1) representa matematicamente o funcionamento do controlador PID:

$$
u(t)=M V(t)=K_{p} e(t)+K_{i} \int_{0}^{t} e(t) d t+K_{d} \frac{d e(t)}{d t}
$$

Onde:

MV - Variável manipulada;

$\mathrm{Kp}$ - Ganho proporcional;

$\mathrm{Ki}$ - Ganho integral;

$\mathrm{Kd}$ - Ganho derivativo.

\subsection{Sintonia do controlador}

Após a instalação do sistema de controle é necessário que seja realizado os ajustes dos parâmetros do controlador PID para que o sistema atue de forma satisfatória. Há vários métodos de sintonia do controlador, onde cada um possui uma determinada característica e, por consequência, passa a ser indicado para certos tipos de sistemas.

\subsubsection{Método Ziegler Nichols}

O primeiro método baseia-se na resposta transitória da planta ao ser aplicado um degrau no sistema em malha aberta.

De acordo com Ogata (2003), caso haja um integrador ou par de pólos complexo conjugado dominante na planta, não será possível a utilização desse método. Portanto, para ser possível o sinal de saída da planta em malha aberta deve ter uma resposta em forma de s, conforme observado na Figura 1.

Figura 1 - Curva de resposta em forma de S

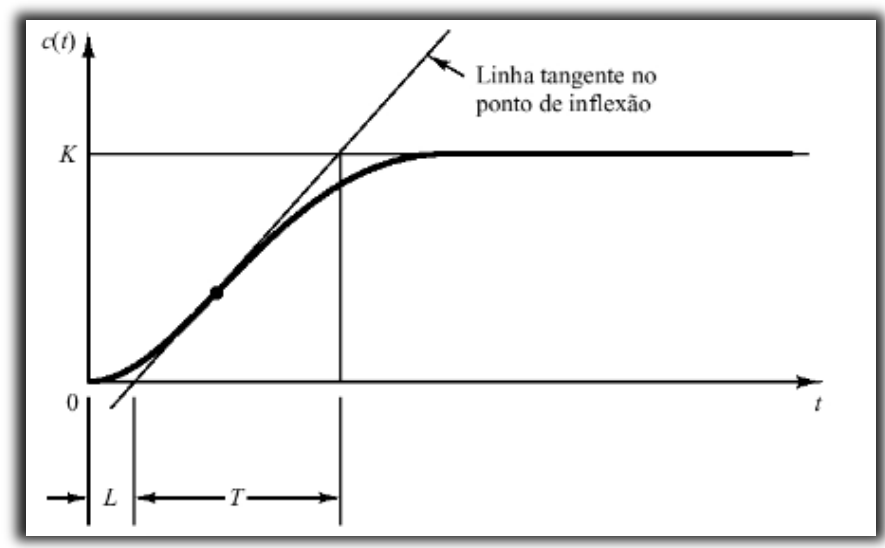

Fonte: OGATA, 2003 
Ao desenhar uma linha reta tangente ao ponto de inflexão da curva com o formato de $S$ podemos encontrar o atraso $L$ do sistema e a constante de tempo $T$, sendo possível determinar a função de transferência desse sistema através da equação 2 de um sistema de primeira ordem com um atraso.

$$
\frac{C(s)}{U(s)}=\frac{K e^{-L s}}{T s+1}
$$

A regra de sintonia de Ziegler-Nichols para a determinação dos ganhos é baseada na resposta ao degrau da planta conforme Tabela 1.

\begin{tabular}{c|c|c|c}
\multicolumn{5}{c}{ Tabela 1 - Regra de sintonia de Ziegler-Nichols - } \\
Primeiro método \\
\hline $\begin{array}{c}\text { Tipo de } \\
\text { controlador }\end{array}$ & $K_{p}$ & $T_{i}$ & $T_{d}$ \\
\hline $\mathrm{P}$ & $\frac{T}{L}$ & $\infty$ & 0 \\
\hline $\mathrm{PI}$ & $0,9 \frac{T}{L}$ & $\frac{L}{0,3}$ & 0 \\
\hline $\mathrm{PID}$ & $1,2 \frac{T}{L}$ & $2 L$ & $0,5 L$ \\
\hline
\end{tabular}

Fonte: OGATA, 2003

O segundo método de Ziegler-Nichols compreende em obter a resposta ao degrau unitário de um sistema em malha fechada utilizando um controlador proporcional em série com a planta.

O modo de verificação de viabilidade desse método consiste em variar o ganho $\mathrm{K}_{\mathrm{p}}$ do controlador proporcional de 0 até o valor crítico $\mathrm{K}_{\mathrm{cr}}$, que consiste no valor ao qual a saída exibe uma oscilação sustentada pela primeira vez. Caso a saída não exiba essa oscilação, o método se aplica.

Para se determinar os parâmetros do controlador PID é necessário obter o valor crítico $\mathrm{K}_{\mathrm{cr}}$ e o período crítico $\mathrm{P}_{\mathrm{cr}}$ pelo estudo do domínio da frequência, podendo assim definir os parâmetros conforme relação da Tabela 2 (LEÃO, 2014).

Tabela 2 - Regra de sintonia de Ziegler-Nichols -

\begin{tabular}{c|c|c|c}
\hline \multicolumn{4}{|c}{ Segundo método } \\
\hline $\begin{array}{c}\text { Tipo de } \\
\text { controlador }\end{array}$ & $\boldsymbol{K}_{\boldsymbol{p}}$ & $\boldsymbol{T}_{\boldsymbol{i}}$ & $\boldsymbol{T}_{\boldsymbol{d}}$ \\
\hline $\mathrm{P}$ & $0,5 K_{c r}$ & $\infty$ & 0 \\
\hline $\mathrm{PI}$ & $0,45 K_{c r}$ & $\frac{1}{1,2} P_{c r}$ & 0 \\
\hline $\mathrm{PID}$ & $0,6 K_{c r}$ & $0,5 P_{c r}$ & $0,125 P_{c r}$ \\
\hline
\end{tabular}

Fonte: OGATA, 2003

\subsubsection{Método de Cohen-Coon}

Esse método utiliza o mesmo princípio do primeiro método de Ziegler-Nichols, consiste em determinar a função de transferência em malha aberta do sistema a ser controlado, aproximando-o por um sistema de primeira ordem com atraso. O método adapta a função de transferência a ser controlada aproximando o gráfico da resposta ao degrau em equação de 1a ordem com elevado tempo morto, isto é, com fator de incontrolabilidade $F_{c}=\theta / \tau \geq 0,3$, em que $\theta$ é o atraso e $\tau$ é a constante de tempo do sistema (GARCIA, 2017). 
A Tabela 3 mostra os ajustes.

Tabela 3: Regra de sintonia de Cohen-Coon

\begin{tabular}{c|c|c|c}
\hline $\begin{array}{c}\text { Tipo de } \\
\text { controlador }\end{array}$ & $\boldsymbol{K}_{\boldsymbol{p}}$ & $\boldsymbol{T}_{\boldsymbol{i}}$ & $\boldsymbol{T}_{\boldsymbol{d}}$ \\
\hline $\mathrm{P}$ & $\frac{1}{K} \cdot\left(\frac{\tau}{\theta}+\frac{1}{3}\right)$ & 0 & 0 \\
\hline $\mathrm{PI}$ & $\frac{1}{K} \cdot\left(\frac{0,9 \tau}{\theta}+\frac{1}{12}\right)$ & $3 \theta \cdot \frac{10+\frac{\theta}{\tau}}{9+\frac{20 \theta}{\tau}}$ & 0 \\
\hline $\mathrm{PD}$ & $\frac{1}{K} \cdot\left(\frac{1,25 \tau}{\theta}+\frac{1}{6}\right)$ & 0 & $2 \theta \cdot \frac{3-\frac{\theta}{\tau}}{22+\frac{3 \theta}{\tau}}$ \\
\hline PID & $\frac{1}{K} \cdot\left(\frac{4 \tau}{3 \theta}+\frac{1}{4}\right)$ & $\theta \cdot \frac{32+\frac{6 \theta}{\tau}}{13+\frac{8 \theta}{\tau}}$ & $\frac{4 \theta}{11+\frac{2 \theta}{\tau}}$ \\
\hline
\end{tabular}

Fonte: GARCIA, 2017

\section{MATERIAIS E MÉTODOS}

O circuito de acionamento da carga pode ser visto na Figura 2. Utilizou-se um resistor de 10 $\Omega / 5 \mathrm{~W}$ como carga de aquecimento, um transistor Mosfet IRF530, resistores de $1 \mathrm{k} \Omega / 1 / 4 \mathrm{~W}$ e um optoacoplador PC817 para isolar o circuito de comando eletrônico (gerador do sinal de chaveamento) do circuito de potência. A fonte utilizada é de $12 \mathrm{~V}$ e $5 \mathrm{~A}$.

Figura 2 - Circuito de acionamento

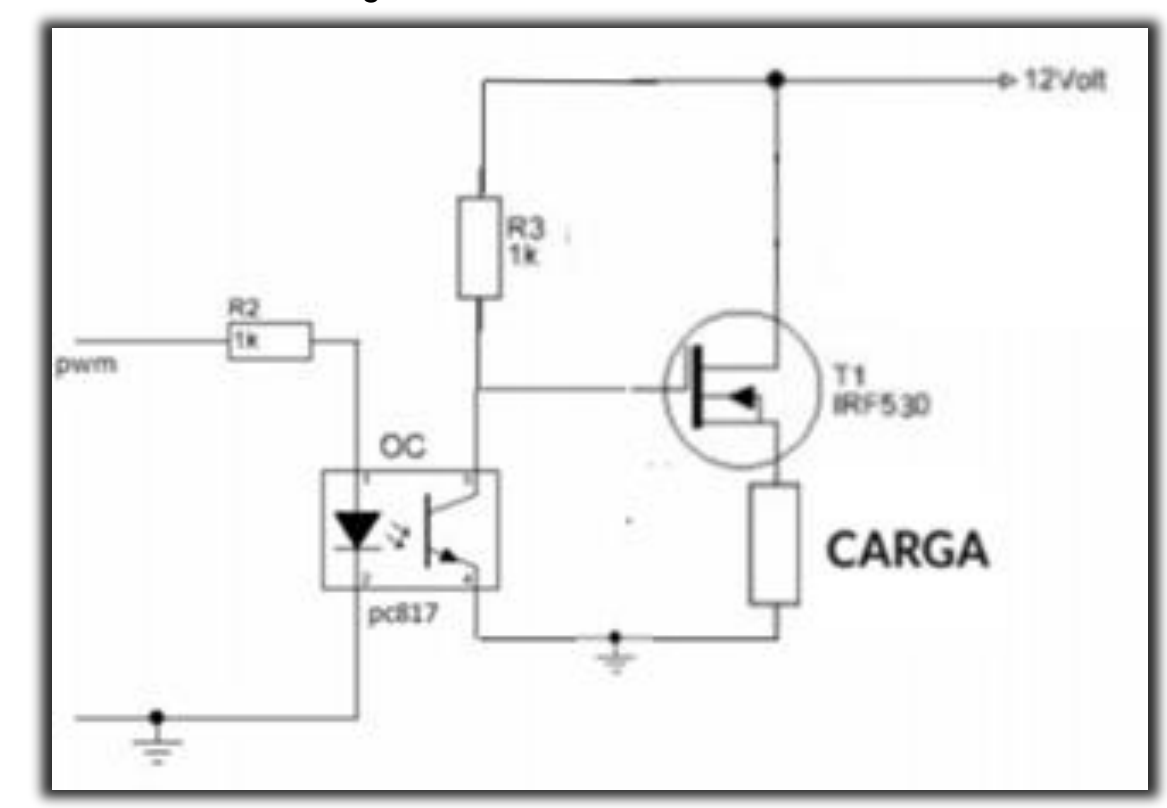

Fonte: Elaborado pelos autores

Para o circuito eletrônico, um Arduino modelo UNO é utilizado como interface de comunicação entre o circuito de acionamento e a malha de controle, desenvolvida no software MATLAB/Simulink. O circuito montado pode ser visto na Figura 3. 
Figura 3 - Circuito montado.

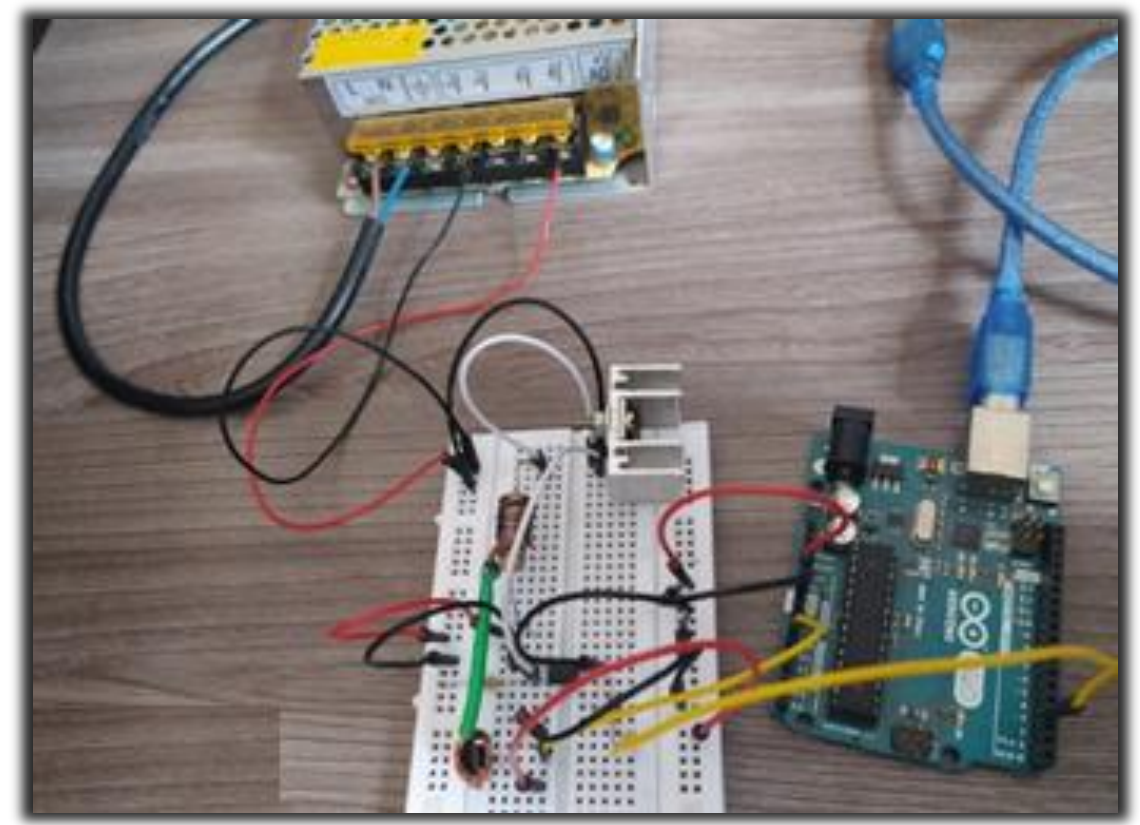

Fonte: Elaborado pelos autores

O Arduino envia o sinal de entrada do circuito (um degrau ou um sinal PWM) e realiza a leitura da temperatura na carga, através de um sensor LM35 acoplado em um fio de cobre de $2,5 \mathrm{~mm}$, com $4 \mathrm{~cm}$ de comprimento. $O$ fio de cobre é utilizado para gerar um atraso de propagação da temperatura.

\section{RESULTADOS E DISCUSSÕES}

A Figura 4 mostra a malha aberta desenvolvida no software MATLAB/Simulink. Um sinal PWM é gerado em script (com amplitude variável de 0 a $5 \mathrm{~V}$ ), convertido e enviado a entrada do optoacoplador (pino 3 do Arduino). A temperatura lida (pino 0 do Arduino) é convertida e projetada em um scope.

Figura 4 - Malha aberta de controle

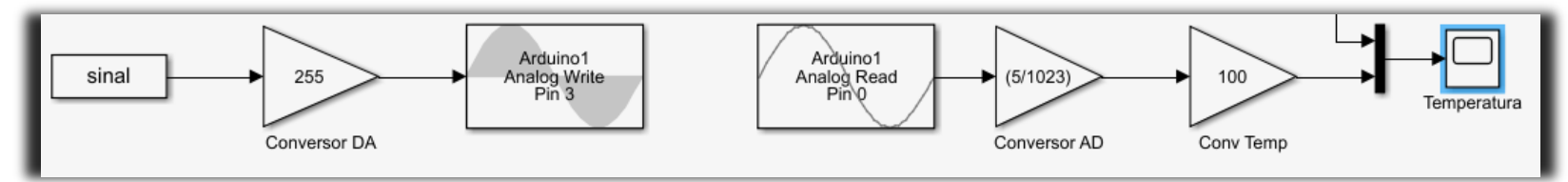

Fonte: Elaborado pelos autores

A Figura 5 mostra a resposta do sistema ao sinal aplicado. O PWM varia de $0 \%$ a $30 \%$ (0s a 400s) no primeiro instante e em sequência (401s a 800 s) de $30 \%$ a $50 \%$. 


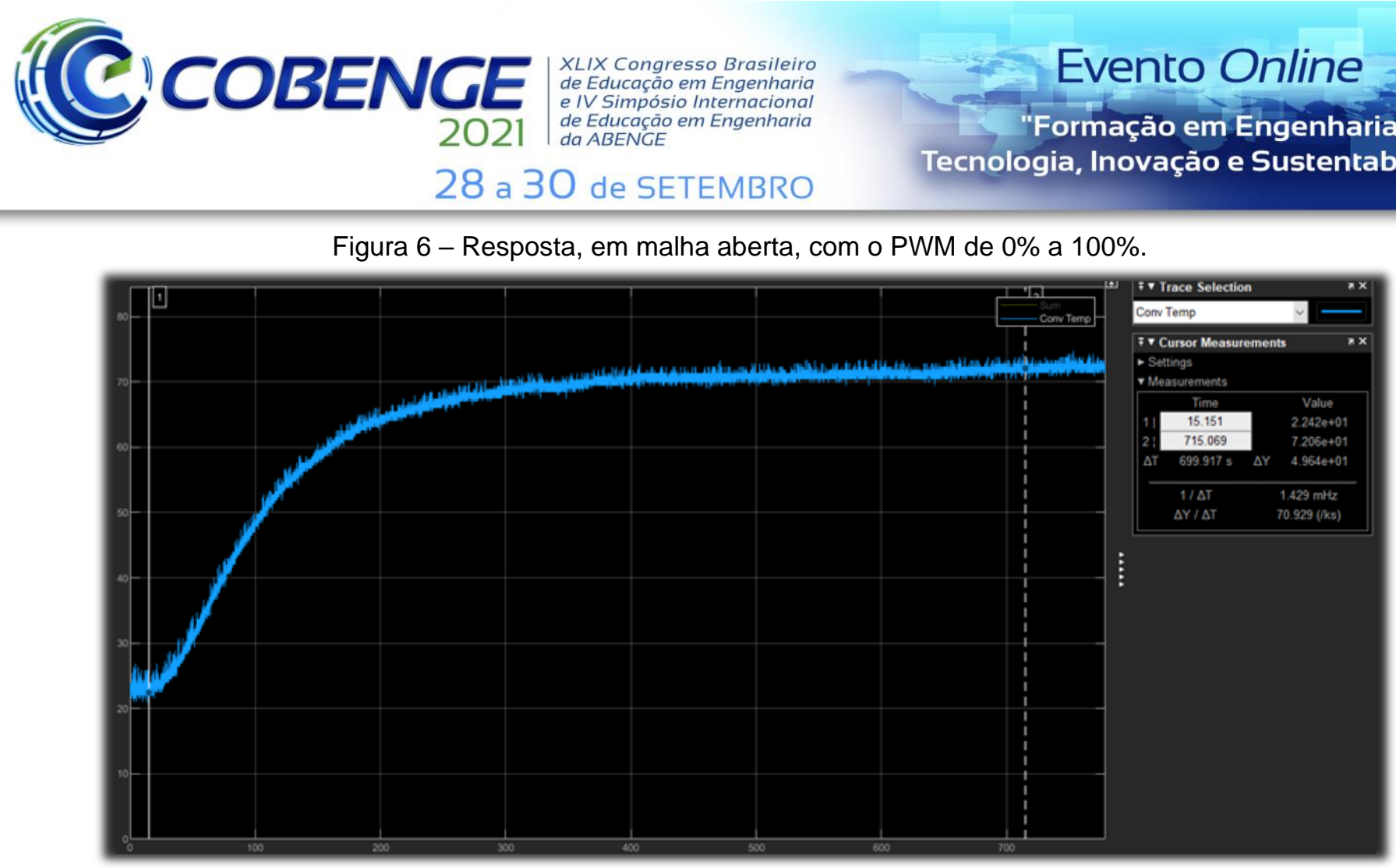

Fonte: Elaborado pelos autores

$$
K_{C \%}=\frac{\Delta L}{\Delta T}=\frac{100-0}{72-23}=2,041\left[\frac{\%}{{ }^{\circ} C}\right]
$$

Assim:

$$
G=\frac{K_{C \%} \cdot K \cdot e^{-\theta s}}{\tau s+1}=\frac{2,041 \cdot 0,983 \cdot e^{-21 s}}{108 s+1}=\frac{2,006 \cdot e^{-21 s}}{108 s+1}[\text { adim } .]
$$

A Figura 7 mostra a resposta do sistema ao sinal aplicado com ênfase ao instante do PWM (401s a 800 s) de $30 \%$ a $50 \%$.

Figura 7 - Resposta do sistema, em malha aberta, com PWM entre $30 \%$ e 50\%.

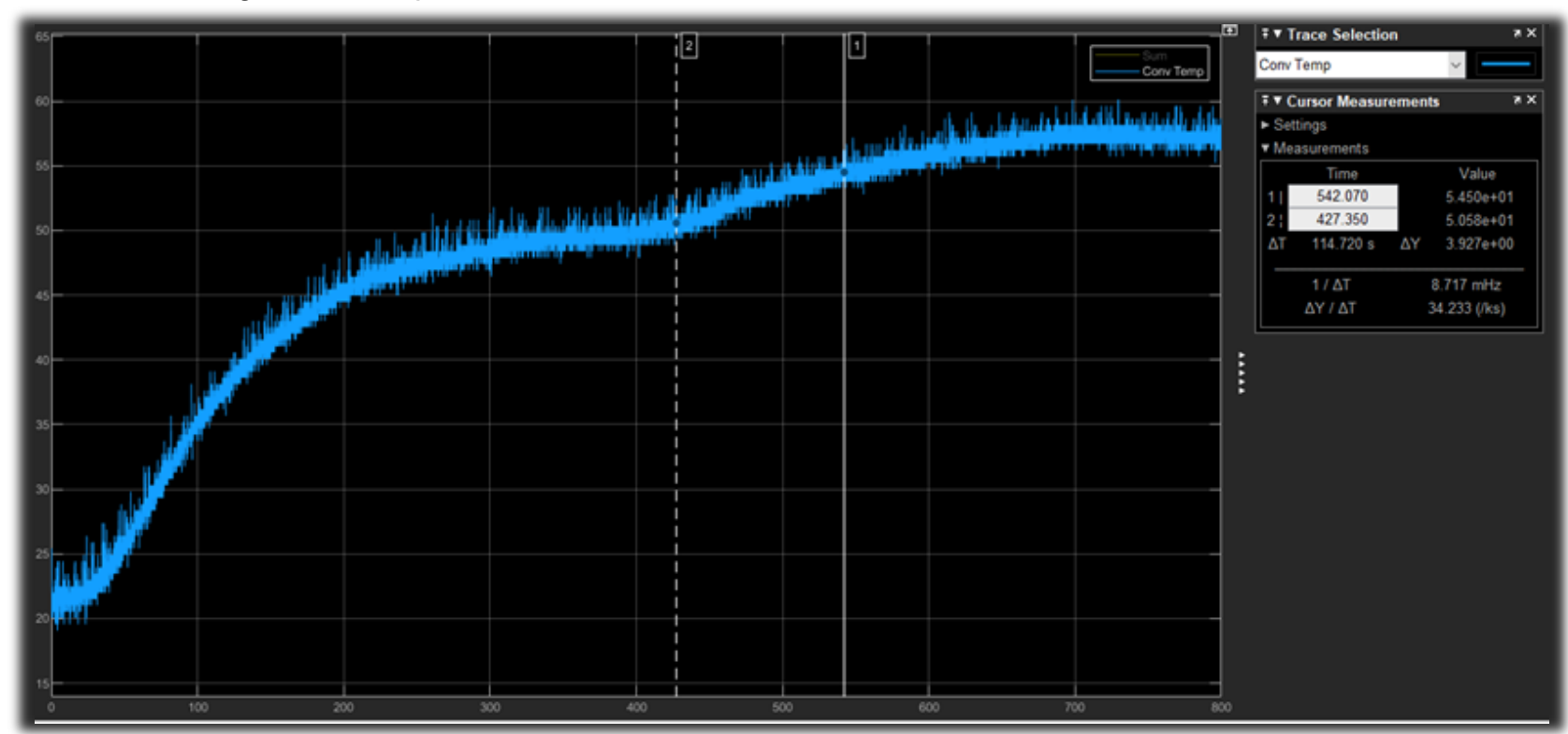

Fonte: Elaborado pelos autores 
Analisando o segundo estágio, obtêm-se:

- Atraso de 27s;

- Temperatura inicial (Ti) igual a $50^{\circ} \mathrm{C}$;

- Temperatura final (Tf) igual a $57^{\circ} \mathrm{C}$;

- Tempo de subida (Ts) igual a 114 segundos.

Nota-se que o sistema possui um comportamento não linear, pois os seus parâmetros, em malha aberta, são distintos.

Calculando-se a função de transferência do sistema para o segundo estágio e realizando o ajuste adimensional, obtêm-se:

$$
G=\frac{K_{C \%} \cdot K \cdot e^{-\theta s}}{\tau s+1}=\frac{2,041 \cdot 0,35 \cdot e^{-27 s}}{114 s+1}=\frac{0,714 \cdot e^{-27 s}}{114 s+1}[\mathrm{adim} .]
$$

\subsection{Projeto e sintonia do controlador}

De acordo com o método de sintonia Cohen-Coon, é aconselhável que o fator de incontrolabilidade (relação entre o atraso $\theta$ e o tempo de subida $\tau$ seja maior ou igual a 0,3 . Para o primeiro estágio tem-se:

$$
F_{C}=\frac{\theta}{\tau}=\frac{21}{108}=0,194
$$

Para o segundo estágio:

$$
F_{C}=\frac{\theta}{\tau}=\frac{27}{114}=0,237
$$

Mesmo com um resultado inferior em ambos os estágios, calculou-se os ganhos do controlador a partir dos dados do estágio 1, conforme a Equação 10 e Equação 11.

$$
\begin{aligned}
& K_{C}=\frac{1}{K} \cdot\left(\frac{0,9 \cdot \tau}{\theta}+\frac{1}{12}\right)=\frac{1}{2,006} \cdot\left(\frac{0,9 \cdot 108}{21}+\frac{1}{12}\right)=2,349 \\
& T_{I}=3 \cdot \theta \cdot\left(\frac{\left(10+\frac{\theta}{\tau}\right)}{9+\frac{20 \cdot \theta}{\tau}}\right)=3 \cdot 21\left(\frac{\left(10+\frac{21}{108}\right)}{9+\frac{20.21}{108}}\right)=49,830
\end{aligned}
$$

A malha de controle fechada pode ser visualizada na Figura 8. 
Figura 8 - Malha de controle fechada.

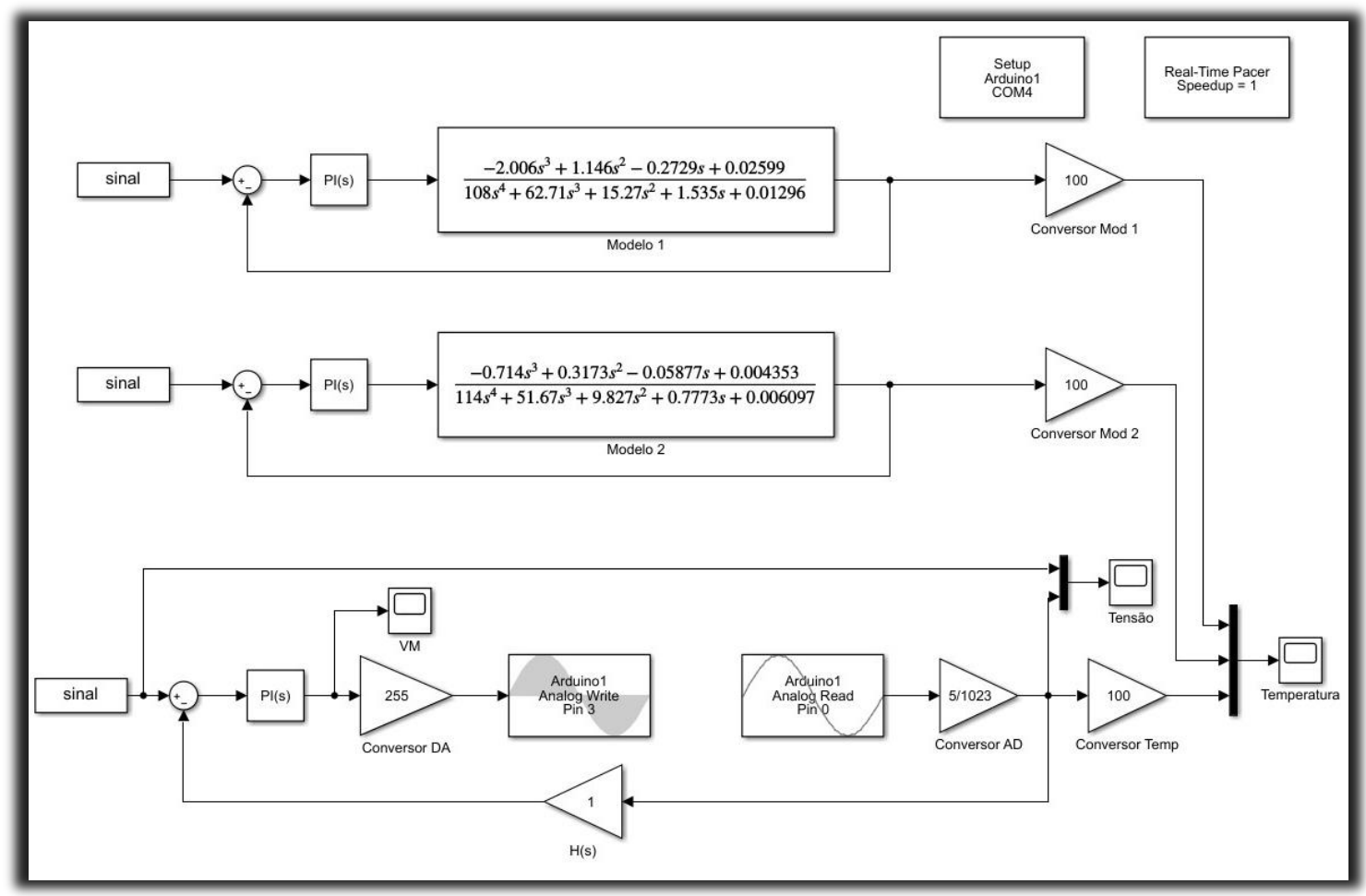

Fonte: Elaborado pelos autores

O modelo 1 representa a identificação do sistema no estágio 1, assim como o modelo 2 representa a identificação do sistema no estágio 2, após realizada a aproximação de Padé de $3^{\mathrm{a}}$ ordem para eliminar a exponencial decrescente das funções de transferência. Por fim tem-se a malha fechada do sistema físico.

A configuração dos parâmetros do controlador PI pode ser vista na Figura 9.

Figura 9 - Parâmetros do controlador.

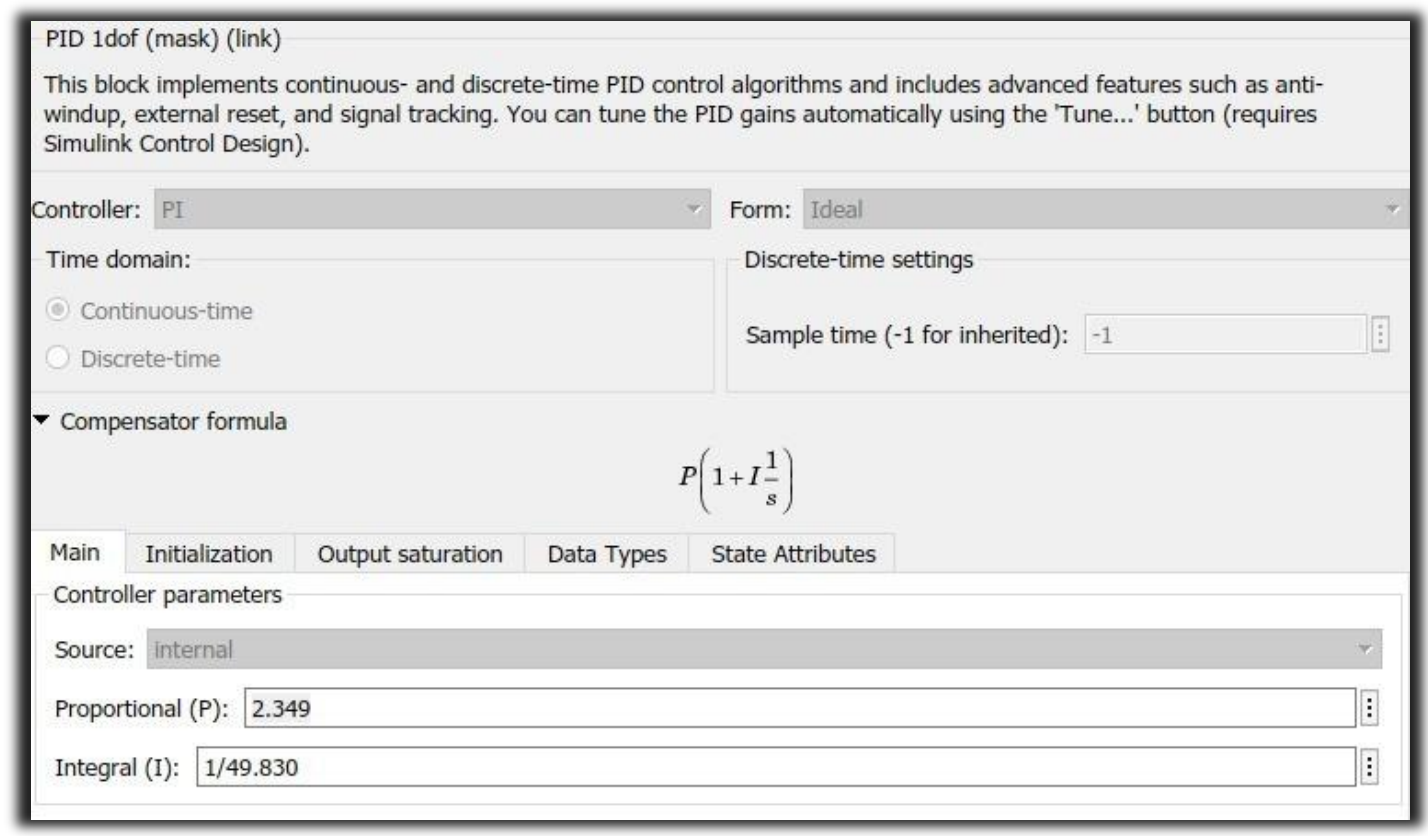

Fonte: Elaborado pelos autores 


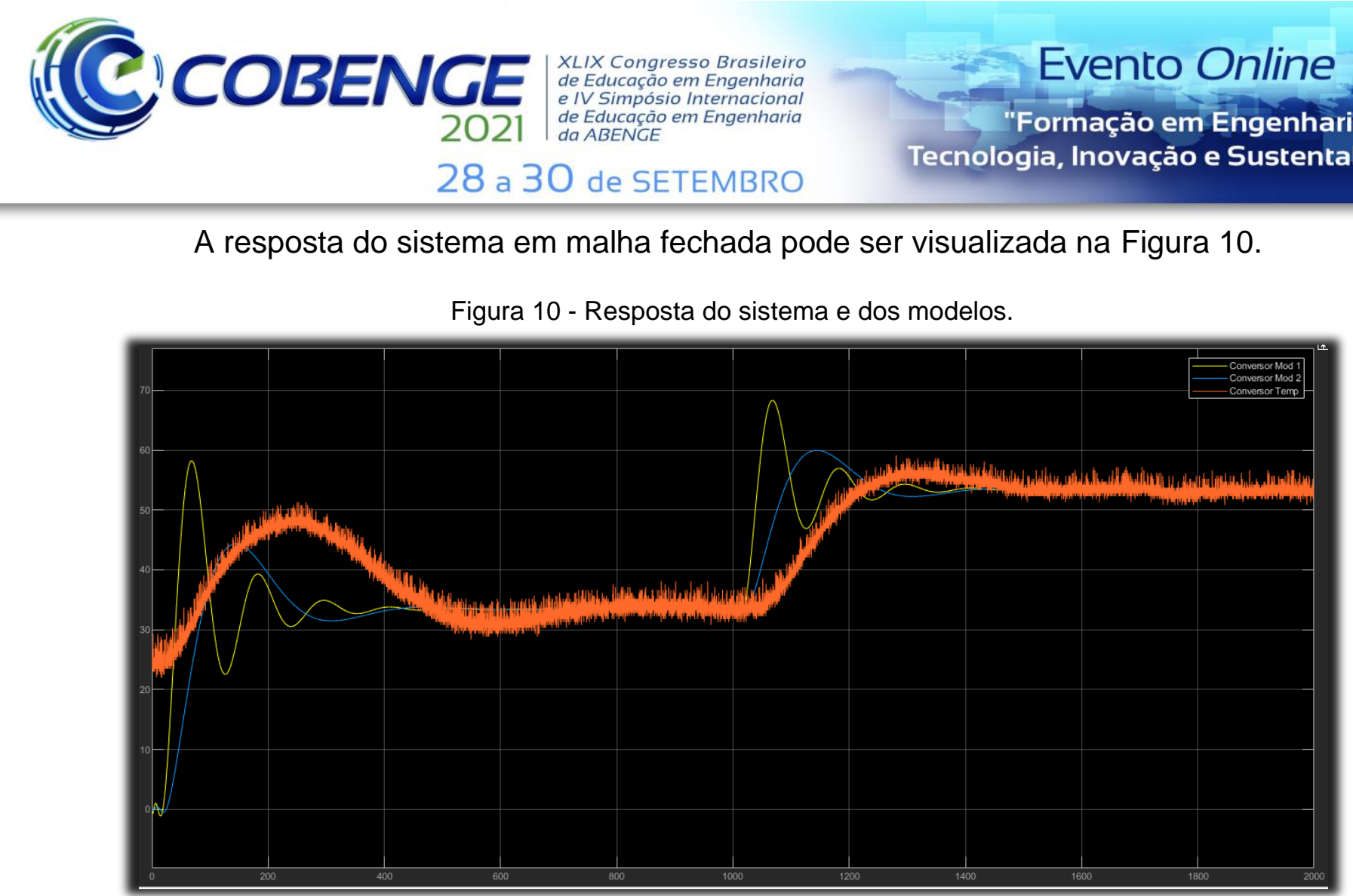

Fonte: Elaborado pelos autores

\section{$5 \quad$ CONSIDERAÇÕES FINAIS}

Nesse projeto procurou-se mostrar a importância da aplicação dos conceitos estudados em teoria de controle no processo de ensino-aprendizagem, comprovando a implementação de um sistema de controle em malha fechada, com atraso de propagação, em uma planta física de fácil construção.

Foram realizados uma série de ensaios com o objetivo de controlar a temperatura de um sistema termoelétrico, utilizando-se de ferramentas computacionais e uma interface eletrônica de baixo custo. A técnica da caixa preta foi utilizada para identificar a função de transferência do sistema e, assim, implementar o controlador.

Na primeira etapa do projeto, o sinal PWM foi variado de $0 \%$ a $30 \%$ e de $30 \%$ a $50 \%$. Observou-se que o sistema não é linear, dado que em cada ensaio realizado, os parâmetros da resposta foram distintos.

Para sintonizar o controlador PI foi utilizado o método Cohen-Coon. O método foi aplicado nos dois modelos obtidos e na planta física, como observado na Figura 10. Em ambas as respostas modeladas, o comportamento transitório foi diferente do observado na planta física, porém, em regime permanente, ambas as respostas coincidem com o sistema real.

O sistema desenvolvido, de simples construção e baixo custo, pode ser utilizado para otimizar as atividades laboratoriais no ensino de engenharia, auxiliando os estudantes na aplicação de conceitos teóricos e permitindo-os enfrentar dificuldades práticas de projeto e controle de uma planta termoelétrica.

Como sugestão de trabalhos futuros, outros métodos de sintonia podem ser aplicados a fim de se obter uma melhor resposta do sistema, bem como diferentes tipos de controladores. 


\title{
REFERÊNCIAS
}

Dixon, W. E. et al. A MATLAB-based control systems laboratory experience for undergraduate students: toward standardization and shared resources. IEEE Transactions on Education, v. 45, n. 3, p. 218-226, 2002, doi: 10.1109/TE.2002.1024613. Disponível em: https://ieeexplore.ieee.org/abstract/document/1024613/. Acesso em: 22 abr.2021

GARCIA, Claudio. Controle de Processos Industriais. Vol. 1. Estratégias Convencionais. São Paulo: Editora Edgard Blucher Ltda, 2017.

GOMES, F. J. et. al. Módulo laboratorial de baixo custo, baseado em FOSS, para Educação em Engenharia de Controle de Processos Industriais. In: XXXIX Congresso Brasileiro de Educação em Engenharia, Cobenge, FURB, 2011, Blumenau. Anais. Disponível em: https://www.ufjf.br/peteletrica/files/2010/03/M\%C3\%B3dulo-Laboratorial-de-Baixo-CustoBaseado-em-FOSS-para-Educa\%C3\%A7\%C3\%A3o-em-Engenharia-de-Controle-deProcessos-Industriais-CLAG.pdf. Acesso em: 24 abr.2021

LEÃO, André Luis de Araújo Marques. Métodos de sintonização automática de controladores PIDs e análise estatística de desempenho. 2014. Trabalho final (Graduação) - Curso de Engenharia de Controle e Automação. Universidade Estadual de Campinas - Faculdade de Engenharia Mecânica, Campinas, 2014. Disponível em: http://www.fem.unicamp.br/ fioravanti/tgs/andre1s14.pdf. Acesso em: 14 abr.2021.

NOVUS. Artigo técnico - controle PID básico. 2003 Disponível em: https://www.novus.com.br/artigosnoticias/arquivos/ArtigoPIDBasicoNovus.pdf. Acesso em: 13 abr. 2021.

OGATA, Katsuhiko. Engenharia de Controle Moderno. 4 ed. São Paulo: Editora PrenticeHall, 2003

\section{DESIGN AND DEVELOPMENT OF AN INDUSTRIAL CONTROLLER APPLIED TO A LOW-COST THERMOELECTRIC SYSTEM}

\begin{abstract}
This work aims to facilitate the teaching-learning process of the disciplines of control theory by applying, in a didactic plant of simple construction and low-cost, techniques of identification, modeling, design and tuning of industrial controllers. The thermoelectric system was designed with discrete components in conjunction with the Arduino platform and the control loop was implemented with the aid of MATLAB / Simulink software. The plant has a propagation delay, which makes it possible to use tuning techniques for the less common PID controller, such as the Cohen-Coon method. The results showed that the identification of the system and the tuning of the controller were satisfactory since the temperature of the models and the physical plant showed a stable behavior in a permanent regime.
\end{abstract}

Keywords: Process control. PID controller. Didactic plant. Thermoelectric system. 